\title{
X-ray-induced conductivity in iron-doped lithium niobate crystals
}

\author{
D. Berben, ${ }^{1}$ B. Sturman, ${ }^{2}$ A. A. Freschi, ${ }^{3}$ and K. Buse ${ }^{1}$ \\ ${ }^{1}$ University of Bonn, Institute of Physics, Wegelerstr. 8, D-53115 Bonn, Germany \\ ${ }^{2}$ Russian Academy of Sciences, Institute for Automation \& Electrometry, Novosibirsk 630090, Russia \\ ${ }^{3}$ Universidade Estadual Paulista, Departamento de Fisica, CP178, 13500-970, Rio Claro, SP, Brazil
}

(Received 4 April 2003; published 31 July 2003)

\begin{abstract}
Illumination of photorefractive, iron-doped lithium niobate crystals $\left(\mathrm{LiNbO}_{3}: \mathrm{Fe}\right)$ with $\mathrm{x}$-rays generates a conductivity that we determine from the speed of hologram erasure. The doping levels of the crystals and the acceleration voltage of our x-ray tube are varied. A theoretical model is presented, which describes the obtained results. A decrease of the conductivity with increasing $\mathrm{Fe}^{2+}$ concentration can be explained by assuming that holes are the dominant charge carriers for this short-wavelength illumination.
\end{abstract}

DOI: 10.1103/PhysRevB.68.035120

\section{INTRODUCTION}

The behavior of photorefractive lithium niobate crystals $\left(\mathrm{LiNbO}_{3}\right)$ upon illumination with visible light has been studied for more than 30 years. ${ }^{1,2}$ In iron-doped material $\mathrm{LiNbO}_{3}: \mathrm{Fe}$, charge carriers are excited from $\mathrm{Fe}^{2+}$ centers and migrate through the crystal due to the bulk photovoltaic effect, ${ }^{3}$ diffusion, and drift. The electrons are preferentially trapped in the darker regions by $\mathrm{Fe}^{3+}$ centers. ${ }^{4}$ Inhomogeneous illumination thus leads to an inhomogeneous distribution of charge carriers, which results in an electric spacecharge field and hence in a refractive-index modulation due to the electro-optic effect. ${ }^{5,6}$

Investigations of the influence of $\mathrm{x}$-ray exposure on the photorefractive properties have been performed by several authors, ${ }^{7-9}$ however, a detailed study of the conductivity of $\mathrm{LiNbO}_{3}: \mathrm{Fe}$ for various doping levels, oxidization/reduction states and radiation energies is missing so far. In this work we investigate in detail the influence of doping level and annealing on the $\mathrm{x}$-ray-induced conductivity. The outcomes are of relevance for an $\mathrm{x}$-ray imaging method that is based on the erasure of elementary holograms by x-rays. ${ }^{10}$ The experiments are performed for several doping levels ranging from 0.005 wt $\% \mathrm{Fe}_{2} \mathrm{O}_{3}$ up to 0.25 wt $\% \mathrm{Fe}_{2} \mathrm{O}_{3}$ and for radiation energies with acceleration voltages from $50 \mathrm{kV}$ up to $120 \mathrm{kV}$.

\section{THEORETICAL CONSIDERATIONS}

The simplest model to describe an x-ray-induced conductivity is presented in Fig. 1. The dominant process for creation of free charge carriers is assumed to be generation of electron-hole pairs by x-ray photons. Basically, any atom inside the crystal can be ionized by photons with energies of several keV. Free electrons and holes can be trapped by $\mathrm{Fe}^{3+}$ or $\mathrm{Fe}^{2+}$, respectively. This yields the following rate equations:

$$
\begin{gathered}
\frac{d n}{d t}=\alpha I_{\mathrm{x}}-\gamma_{\mathrm{n}} n N^{+}, \\
\frac{d p}{d t}=\alpha I_{\mathrm{x}}-\gamma_{\mathrm{p}} p N .
\end{gathered}
$$

PACS number(s): 72.40.+w

Here, $n$ is the density of free electrons, $p$ is the density of free holes, $t$ is the time, $\alpha$ is a coefficient that is proportional to the absorption for the incident $\mathrm{x}$-ray light, $I_{\mathrm{x}}$ is the intensity of the x-ray illumination, $\gamma_{\mathrm{n}}$ and $\gamma_{\mathrm{p}}$ are recombination rates, and $N$ and $N^{+}$are the concentrations of charged and discharged dopant defects, respectively, namely, $N=c_{\mathrm{Fe}^{2+}}$ and $N^{+}=c_{\mathrm{Fe}^{3+}}$. Since the relative number of $\mathrm{Fe}$ atoms in the crystal is small and their atomic weight is not large as compared with that of $\mathrm{Nb}$ atoms, absorption coefficient $\alpha$ does not depend on $N$ or $N^{+}$. Since the concentration of free electrons and holes is much smaller than typical doping concentrations, ${ }^{11}$ the electron-hole recombination of free electrons and holes can be neglected (this rate is proportional to $n p$ which is much less than $n N^{+}$and $p N$ ). In the steadystate case this simplifies to

$$
\begin{gathered}
n=\frac{\alpha I_{\mathrm{x}}}{\gamma_{\mathrm{n}} N^{+}}, \\
p=\frac{\alpha I_{\mathrm{x}}}{\gamma_{\mathrm{p}} N} .
\end{gathered}
$$

Since conductivity $\sigma$ is determined by the concentration and mobility of charge carriers, we can write

$$
\sigma=e\left(\mu_{\mathrm{n}} n+\mu_{h} p\right)=e \alpha I_{\mathrm{x}}\left(\frac{\mu_{\mathrm{n}}}{\gamma_{\mathrm{n}} N^{+}}+\frac{\mu_{\mathrm{p}}}{\gamma_{\mathrm{p}} N}\right),
$$

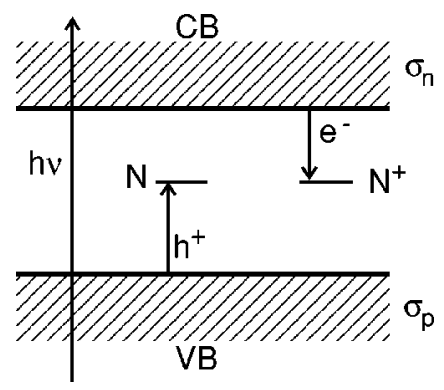

FIG. 1. Band model for the $\mathrm{x}$-ray-induced charge transport. High-energy x-ray photons $h \nu$ can excite charge carriers from any atom inside the crystal, and hence generate electron-hole pairs. Electrons and holes can be trapped by dopants, e.g., $\mathrm{Fe}^{3+}$ and $\mathrm{Fe}^{2+}$ in $\mathrm{LiNbO}_{3}: \mathrm{Fe}\left(N^{+}, N\right.$ : concentrations of $\mathrm{Fe}^{3+}$ and $\left.\mathrm{Fe}^{2+}\right)$. 


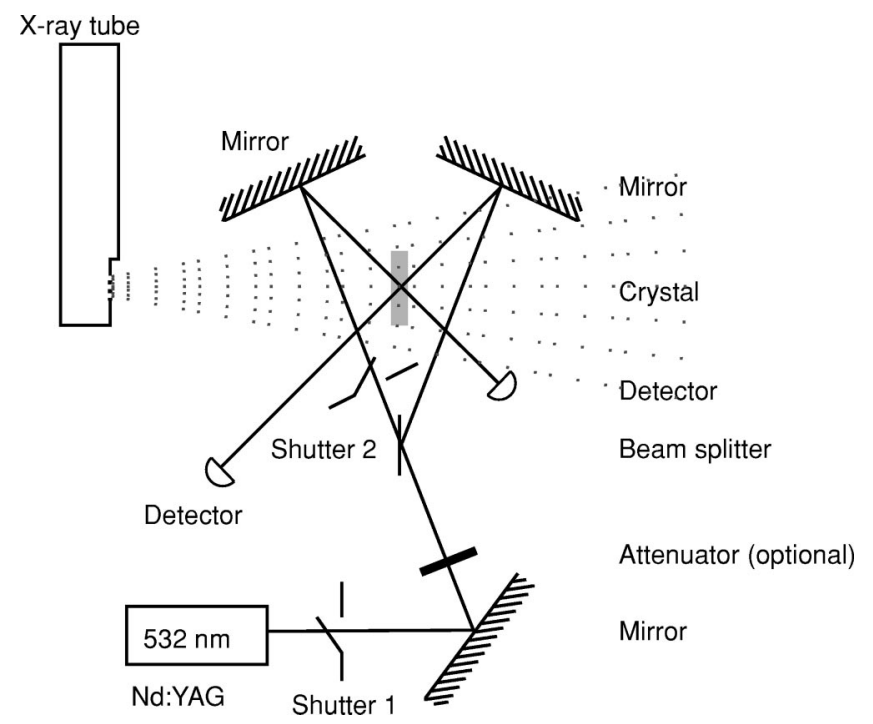

FIG. 2. Experimental setup for recording and reading of holographic reflection gratings. The beam of a frequency-doubled $\mathrm{Nd}$ :YAG laser (wavelength $532 \mathrm{~nm}$ ) is split into two beams of equal power, which are superimposed onto a $\mathrm{LiNbO}_{3}$ crystal. For reading the hologram, shutter 2 is closed and the optional attenuator is placed in the optical path.

where $e$ is the elementary charge, and $\mu_{\mathrm{n}}$ and $\mu_{\mathrm{h}}$ are the electron and hole mobilities, respectively. As seen from this formula, we expect that the x-ray-induced conductivity depends on the sum of two terms which are proportional to $1 / c_{\mathrm{Fe}^{2+}}$ and $1 / c_{\mathrm{Fe}^{3+}}$, respectively. The influence of $N^{+}$ should be negligible if the charge transport is dominated by holes. In this case, the hologram erasure time constant $\tau$ $=\epsilon \epsilon_{0} / \sigma$ should increase linearly with $N=c_{\mathrm{Fe}^{2+}}$. Here, $\epsilon$ and $\epsilon_{0}$ denote the static dielectric constant and the permittivity of free space, respectively.

\section{EXPERIMENTAL METHODS}

Our experimental setup is shown in Fig. 2. An expanded beam of a frequency-doubled $100 \mathrm{~mW}$ single-frequency Nd:YAG laser (wavelength $532 \mathrm{~nm}$ ) is split into two ordinarily polarized beams of equal power, which are superimposed onto the crystal. The total recording intensity is about $160 \mathrm{~mW} / \mathrm{cm}^{2}$. Typical crystal dimensions are $\mathrm{a} \times \mathrm{b} \times \mathrm{c}=14$ $\times 12 \times 0.85 \mathrm{~mm}^{3}$. Reflection geometry with an external angle of $133^{\circ}$ between the two beams is chosen, which corresponds to a grating period length $\Lambda$ of $117 \mathrm{~nm}$ inside the crystal. The crystal is slanted by about $1^{\circ}$ to separate the diffracted beam from the beam that is reflected from the illuminated crystal surface. To avoid buildup of uncontrolled homogeneous electric fields that result from the bulk photovoltaic effect, the crystal is placed inside a plexiglass holder which allows short circuiting of the crystal by liquid electrodes of purified water.

As the radiation source, a dc x-ray tube with tungsten anode and a beryllium window is utilized. Acceleration voltage $U_{\mathrm{a}}$ is adjustable from 50 to $150 \mathrm{kV}$ with currents ranging from 0 to $20 \mathrm{~mA}$. The crystal is placed at a distance of $25 \mathrm{~cm}$ from the focal spot of the $\mathrm{x}$-ray tube without any collimators in the radiation path to ensure a homogeneous $\mathrm{x}$-ray illumination. Lead radiation shields ensure that no $\mathrm{x}$-ray light enters the detectors.

Hologram recording is performed at room temperature until saturation of the diffraction efficiency is reached. The crystal is then removed from the plexiglass holder and put into an oven at a temperature of $165^{\circ} \mathrm{C}$ for one hour to achieve thermal fixing of the hologram. ${ }^{1,12}$ After cooling back to room temperature, the crystal is remounted into the plexiglass holder and the grating is developed using a white light tungsten lamp. For x-ray erasure experiments one beam is blocked by shutter 2 , and the remaining beam that enters the crystal is attenuated to an intensity of only $2 \mathrm{~mW} / \mathrm{cm}^{2}$ to avoid influences of the readout light on the hologram. During the experiment, diffraction efficiency $\eta$ is measured every $120 \mathrm{~s}$ for approximately $0.5 \mathrm{~s}$. Here $\eta$ is defined as $I_{\mathrm{d}} /\left(I_{\mathrm{d}}\right.$ $+I_{\mathrm{t}}$ ), where $I_{\mathrm{d}}$ and $I_{\mathrm{t}}$ are the intensities of the diffracted and transmitted beams. From the value of $\eta$ we deduce, with Kogelniks formula, the amplitude of refractive-index grating $\Delta n$. After each x-ray erasure experiment, the crystal is illuminated with white light for several hours to redevelop the thermally fixed grating in order to guarantee identical starting conditions for each x-ray erasure measurement. For a thermally fixed grating the amplitude of the developed grating is given by

$$
\Delta n \propto E_{\mathrm{sc}} \approx \frac{j_{\mathrm{phv}}}{\sigma_{\mathrm{ph}}},
$$

where $E_{\mathrm{sc}}$ is the space-charge field amplitude, $j_{\mathrm{phv}}$ is the modulated bulk photovoltaic current density, and $\sigma_{\mathrm{ph}}$ is the homogeneous conductivity. X-ray illumination erases $E_{\mathrm{sc}}$ and $\Delta n$ because ratio $j_{\mathrm{phv}} / \sigma_{\mathrm{ph}}$ is several orders of magnitude smaller than that for visible light. However, homogeneous illumination with visible light builds up the original refractive-index changes because a modulated $\mathrm{Fe}^{2+}$ concentration is still present, which creates again a modulated current $j_{\text {phv }}$. Since the concentration of ions which are mobilized during thermal fixing is static at room temperature, this erasure/development process can be repeated many times without fading of the grating.

\section{EXPERIMENTAL RESULTS}

A typical result of an x-ray erasure experiment is shown in Fig. 3. A thermally fixed and fully developed hologram with an initial refractive-index modulation $\Delta n_{0}$ of 3.5 $\times 10^{-5}$ is erased by $\mathrm{x}$-ray radiation with an acceleration voltage of $100 \mathrm{kV}$ and a current of $10 \mathrm{~mA}$. The dashed line is a stretched-exponential fit according to

$$
\Delta n=\Delta n_{0} \exp \left[\left(\frac{t}{\tau}\right)^{\beta}\right]
$$

where $\tau$ is the erasure time constant. All measured curves could be fitted using this stretched-exponential approach with values for stretching exponent $\beta$ ranging from 0.5 to 1 . The values of $\tau$ are ranging from $1.500 \mathrm{~s}$ to more than $100.000 \mathrm{~s}$ in different crystals. 


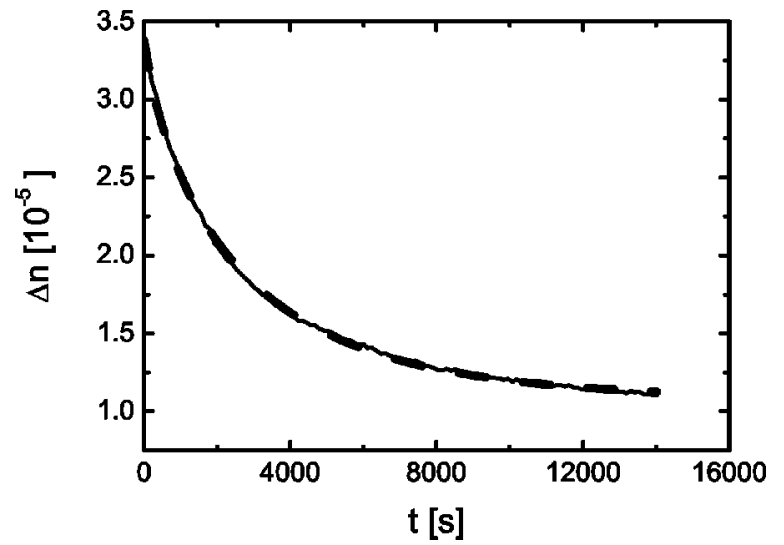

FIG. 3. Typical erasure behavior for a thermally fixed grating for homogeneous x-ray exposure. The initial refractive-index modulation of $3.5 \times 10^{-5}$ is erased with an erasure time constant of roughly $2500 \mathrm{~s}$. The stretched-exponential fit is plotted as a dashed line.

A typical dependence of erasure time constant $\tau$ on the $\mathrm{Fe}^{2+}$ concentration is shown in Fig. 4. The plotted straight line is a linear fit; it shows a good agreement with the experimental data. The slope of this line has the meaning of a specific erasure time which shows the dependence of erasure time $\tau$ on the $\mathrm{Fe}^{2+}$ concentration for a given acceleration voltage. In the following, this slope (measured in $\mathrm{s} \mathrm{m}^{3}$ ) will be denoted by $s^{*}$. A similar presentation of erasure time $\tau$ versus the $\mathrm{Fe}^{3+}$ concentration yields no recognizable pattern.

Slope $s^{*}$ can be regarded as inverted sensitivity $S_{\mathrm{x}}$ for X-ray hologram erasure. Our experimental data on $S_{\mathrm{x}}$ are shown in Fig. 5 for different acceleration voltages $U_{\mathrm{a}}$. A drastic increase of $S_{\mathrm{x}}$ for high acceleration voltages is obvious.

As expected, there is no decay of the strength of the thermally fixed holograms even after many cycles of x-ray erasure and subsequent redevelopment. Upon illumination with white light the diffraction efficiency of every fixed hologram is fully restored to its original value.

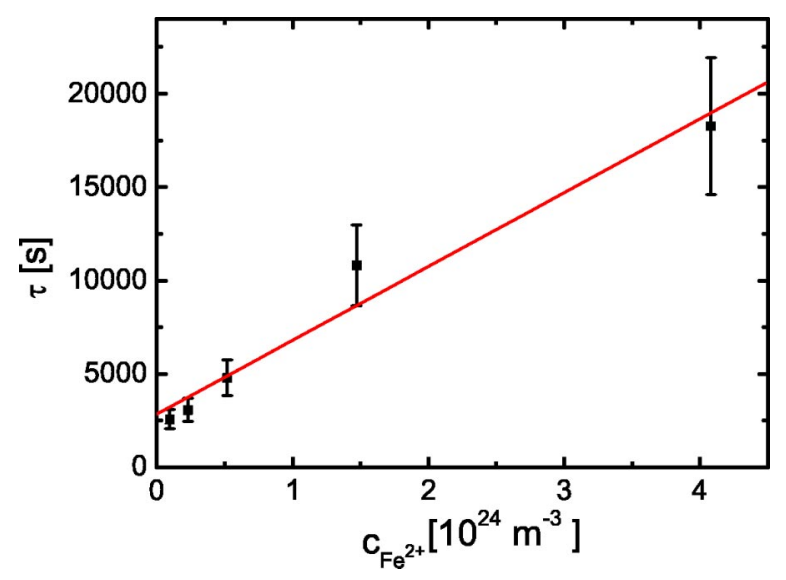

FIG. 4. Decay time constant $\tau$ vs $\mathrm{Fe}^{2+}$ concentration for an acceleration voltage $U_{\mathrm{a}}$ of $100 \mathrm{kV}$ and a current of $10 \mathrm{~mA}$. The line is a linear fit.

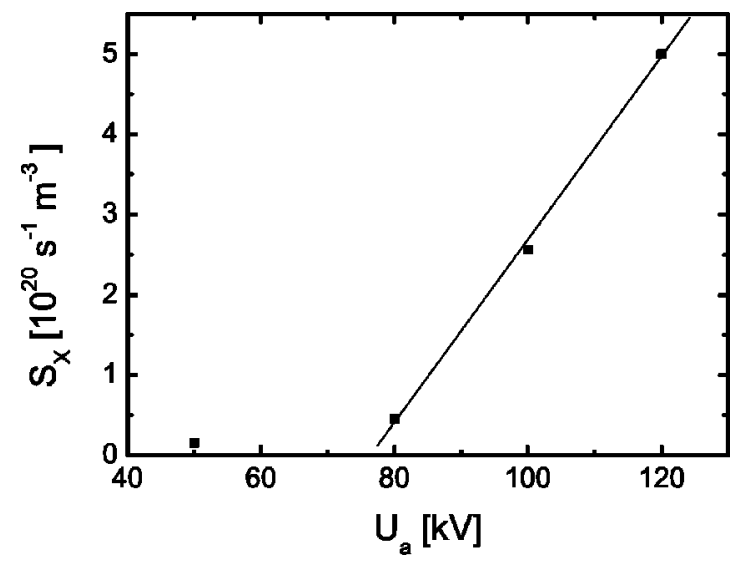

FIG. 5. Value of sensitivity $S_{\mathrm{x}}$ of the x-ray-induced erasure vs acceleration voltage $U_{\mathrm{a}}$. The line is a guide to the eye.

\section{DISCUSSION}

The stretched-exponential behavior of the erasure curves can be attributed to two factors. First, high attenuation coefficients of the soft part of the $\mathrm{x}$-ray radiation provide a nonuniform distribution of x-ray intensity along the beam propagation, causing a nonuniformity of erasure times. Due to the high content of $\mathrm{Nb}$, the absorption for $\mathrm{x}$-ray radiation of less than $40 \mathrm{keV}$ is rather high with a 1/e depth of less than $200 \mu \mathrm{m}$. Second, $\mathrm{x}$-ray illumination of $\mathrm{LiNbO}_{3}: \mathrm{Fe}$ leads to strong coloration which is due to formation of additional $\mathrm{Fe}^{2+} .{ }^{13}$ Thus, the $\mathrm{Fe}^{2+}$ concentration in Eq. (5) is not constant, but changes during the x-ray illumination until it reaches saturation. With the conductivity changing over time, the erasure speed also changes, which leads to nonexponential hologram decay.

The remaining portion of the hologram, which is not erased by $\mathrm{x}$-ray illumination within a reasonable time, may result from the limited penetration depth of the $\mathrm{x}$-ray light used in $\mathrm{LiNbO}_{3}$. This allows part of the hologram to persist in the rear part of the crystal. This remaining hologram can eventually be erased by much longer or higher energetic $\mathrm{x}$-ray illumination.

All holograms have been erased by x-ray radiation many times and were always redeveloped to their original strength; this clearly indicates that the ionic grating, which is formed during the thermal fixing process, is not harmed by $\mathrm{x}$-ray radiation.

Since the erasure of holograms utilizing x-ray illumination is several orders of magnitude faster for strongly oxidized samples (small $\mathrm{Fe}^{2+}$ content), we have focused our attention on oxidized rather than on reduced crystals. In our experiments we observe that the erasure time depends linearly on the $\mathrm{Fe}^{2+}$ concentration and hence the x-ray-induced conductivity depends reciprocially on the $\mathrm{Fe}^{2+}$ content. As it follows from Eq. (5), the $1 / c_{\mathrm{Fe}^{2}}+$ behavior of the conductivity strongly indicates a charge transport which is dominated by holes instead of electrons. This is in good agreement with the early work of Orlowski and Krätzig, ${ }^{14}$ who reported that holes become the dominant charge carriers in $\mathrm{LiNbO}_{3}$ : Fe for highly oxidized samples under ultraviolet illumination.

Equation (5) yields an infinite conductivity value and hence predicts very small erasure time constants if $\mathrm{N}\left(\mathrm{Fe}^{2+}\right.$ 
concentration) approaches zero. However, in the experiment (Fig. 4) an offset is observed. This may originate from the above-mentioned formation of $\mathrm{x}$-ray induced $\mathrm{Fe}^{2+}$, which makes it impossible to preserve a small $\mathrm{Fe}^{2+}$ concentration under x-ray illumination. Also, the nonexponential decay, i.e., persistence of the rear part of the hologram, may enlarge the erasure time constant for oxidized samples and low acceleration voltages.

The results obtained in this work show clearly which crystals are ideally suited to improve the sensitivity of a recently presented $\mathrm{x}$-ray sensor that is based on erasure of elementary holograms. ${ }^{10}$ Highly doped and oxidized crystals are clearly preferred. Based on the outcomes of this work we estimate that the required exposure dose of the sensor might be reduced from 90 Gy to around 10 Gy by using optimized $\mathrm{LiNbO}_{3}:$ Fe crystals.

\section{CONCLUSION}

We have investigated the $\mathrm{x}$-ray-induced conductivity in iron-doped $\mathrm{LiNbO}_{3}$ crystals using x-ray erasure of thermally fixed holograms. A theoretical model that assumes electronhole-pair generation by $\mathrm{x}$-ray photons and trapping of the holes by $\mathrm{Fe}^{2+}$ ions explains the dependence of the hologram erasure time constant on the $\mathrm{Fe}^{2+}$ concentration, assuming that holes dominate the x-ray-induced conductivity. For acceleration voltages above $80 \mathrm{kV}$, erasure is strongly enhanced. Optimized crystals that show a fast erasure are excellent candidates for high-energy x-ray imaging.

\section{ACKNOWLEDGMENTS}

The authors thank A. Hoffmann, K. Peithmann, E. Soergel, and T. Volk for fruitful discussions. A. A. Freschi thanks the Fundação de Amparo a Pesquisa do Estado de São Paulo, Brazil (Grant No. 97/13231-6) and Conselho Nacional de Desenvolvimento Cientifico e Tecnologico, Brazil (Grant No. 540294/01-2). Financial support by the Deutsche Forschungsgemeinschaft (project BU 913/8-1) and by the Deutsche Telekom AG is gratefully acknowledged.
${ }^{1}$ A. Ashkin, G.D. Boyd, J.M. Dziedzic, R.G. Smith, A.A. Ballman, J.J. Levinstein, and K. Nassau, Appl. Phys. Lett. 9, 72 (1966).

${ }^{2}$ F.S. Chen, J.T. LaMacchia, and D.B. Fraser, Appl. Phys. Lett. 13, 223 (1968).

${ }^{3}$ A.M. Glass, D. von der Linde, and T.J. Negran, Appl. Phys. Lett. 25, 233 (1974).

${ }^{4}$ H. Kurz, E. Krätzig, W. Keune, H. Engelmann, U. Gonser, B. Dischler, and A. Räuber, Appl. Phys. 12, 355 (1977).

${ }^{5} \mathrm{P}$. Günter, Photorefractive Effects and Materials-Topics in Applied Physics: Photorefractive Materials and Their Applications $I$, edited by P. G̈̈nter and J.-P. Huignard (Springer-Verlag, Berlin, Heidelberg, 1988), Vol. 61, pp. 7-73, 195-236.

${ }^{6}$ K. Buse, Appl. Phys. B: Lasers Opt. B64, 273 (1997); B64, 391 (1997).
${ }^{7}$ T.R. Volk, S.A. Shramchenko, and L.A. Shuvalov, Ferroelectr. Lett. Sect. 2, 55 (1984).

${ }^{8}$ G. Dalba, Y. Soldo, F. Rocca, V.M. Fridkin, and Ph. Sainctavit, Phys. Rev. Lett. 74, 988 (1995).

${ }^{9}$ Y. Ohmori, M. Yamaguchi, K. Yoshino, and Y. Inuishi, Jpn. J. Appl. Phys. 16, 181 (1977).

${ }^{10}$ D. Berben, B. Andreas, and K. Buse, Appl. Phys. Lett. 81, 1567 (2002).

${ }^{11}$ K. Buse, Appl. Phys. B: Lasers Opt. B64, 273 (1997).

${ }^{12}$ K. Buse, S. Breer, K. Peithmann, S. Kapphan, M. Gao, and E. Krätzig, Phys. Rev. B 56, 1225 (1997).

${ }^{13}$ T.R. Volk, S.A. Shramchenko, L.A. Shuvalov, and V.M. Fridkin, Ferroelectr. Lett. Sect. 3, 23 (1984).

${ }^{14}$ R. Orlowski and E. Krätzig, Solid State Commun. 27, 1351 (1978). 